\title{
Screening for hereditary bowel cancer in New Zealand
}

As a histopathologist, albeit with a special interest in gastrointestinal pathology, I am not qualified to provide an overview of the practice of gastroenterology in New Zealand. My involvement, however, in the establishment of a registry to coordinate the treatment of hereditary bowel cancer has certainly led me away from the comfort and security of the microscope into the world of clinical gastroenterology. I arrived in New Zealand in 1988, much impressed by the pioneering work of Jukka-Pekka Mecklin in Finland. I was certain that the epidemiological-clinicalpathological approach to research and practice (for which the Scandinavian countries can take so much credit) could be emulated in the Dante-esque earthly paradise of the Antipodes. New Zealand has a small and comparatively stable population of a little over three million making it ideal for epidemiologically based research. The decision to investigate bowel cancer in a country with one of the highest rates in the world (and still increasing) seemed obvious at the time. The incidence is considerably lower in the Maori and Polynesian inhabitants of New Zealand, for reasons that are still obscure. On the other hand, inherited colorectal cancer syndromes such as familial adenomatous polyposis are associated with similar gene frequencies in high and low risk populations for colorectal cancer. To facilitate the difficult process of securing funds for research, I decided to concentrate on hereditary nonpolyposis colorectal cancer (HNPCC) (Lynch syndrome), comparatively little being known of its frequency, pathology, optimum treatment or even whether it was truly a single gene disease.

In retrospect, my decision to work on HNPCC was naive and I soon came up against a formidable set of barriers. Although colorectal cancer has turned out to be one of the most important models for studying the molecular genetics of solid tumours, it did not feature as one of the research priorities in New Zealand at the time of my first application for funding. Furthermore, despite the absolute necessity for establishing a registry as the starting point for the treatment of disorders such as HNPCC, I suspect the difficulty in convincing either granting bodies or health managers that this is so is universal. I was new on the scene, however, and a small seeding grant started the ball rolling. The first barrier was down.

New Zealand used to have 14 area health boards, hence 14 ethics committees. A second barrier was the prospect of negotiating with 14 different committees. We feared that each committee would require its own version of the consent form and information sheet and the members of the same family living in various parts of New Zealand would have to receive different documentation according to the decree of their local ethics committee. Fortunately, common sense prevailed and Auckland was accepted as the 'parent' committee. As soon as this barrier was overcome to the satisfaction of all 14 ethical committees, the area health boards, and their ethical committees were dissolved. Mercifully, the arrangement whereby Auckland would serve as the parent committee was transferred to the smaller number of regional ethics committees.

The registry for HNPCC was established in 1989 and from the outset we adopted the strict policy of verifying all diagnoses, including full histopathological confirmation and recording of information according to standardised protocols. The goodwill and graciousness of my histopathological colleagues throughout New Zealand (and overseas) made this possible. One reason for this approach was to be certain of our facts before inviting families to participate in the
HNPCC Registry. The third barrier to be faced was the introduction of the privacy code (based on the Privacy Act), which (from my biased perspective) meant that medical records officers were excused from obtaining the records of deceased patients, unless I could obtain the fully informed consent of the next of kin. This will be a difficult problem to resolve.

HNPCC is a family problem and families do not necessarily live within one country, let alone region or area. This problem is now compounded by the health reforms occurring in New Zealand (paralleling those in the United Kingdom) that are compartmentalising the provision of health care delivery in a way that is compromising the transfer of information and specimens from one hospital to another (the fourth barrier). (It may no longer be politically correct to use the word 'hospital'. We have crown health enterprises, or CHEs, as exact equivalents of the Trusts of the UK.)

One of the most important roles of the HNPCC Registry is to coordinate a nationwide colonoscopic screening programme. Over 400 at risk subjects have now had a colonoscopy and $15 \%$ have had either adenoma or carcinoma. This programme and the collation of colonoscopic findings would not have been possible without the support of gastroenterologists and surgeons throughout New Zealand. A fifth barrier appeared, however, in the form of charges for colonoscopy in the public sector, the charge varying considerably from one $\mathrm{CHE}$ to another. The compliance rate for colonoscopic screening will probably drop from the present high level of $90 \%$ as a result of these charges. Should there be a charge at all for screening of this high risk group of subjects? My fear that the CHE would want full cost recovery for colonoscopy from research funds (this sixth barrier would have been the end of the story) proved groundless. Colonoscopy was accepted as a clinically rather than a research driven investigation.

It is important that clinical geneticists participate in the treatment of hereditary disorders such as HNPCC. Essential backup for a clinical geneticist is a National Genetics Registry and a Centre for Genetics Testing. New Zealand lacks a National Genetics Registry and Centre for Genetics Testing and almost lacks a clinical geneticist (at the time of writing there is one in Wellington). The exponential growth of knowledge in the field of clinical genetics, the value of genetic testing, the psychological impact of testing, and the need for appropriate support and counselling have exposed New Zealand's major deficiency in this area, the seventh barrier.

Despite the seven barriers, great progress has been made in the fields of both research and practice. The registry has shown its ability to coordinate a colorectal cancer prevention programme, a New Zealand family participated in the search that led to the discovery of the first HNPCC gene (hMSH2) on chromosome 2, $80 \mathrm{HNPCC}$ families have been registered, much has been learnt of the natural history of HNPCC, and genetic testing has begun. There are few countries in the world in which so much could have been accomplished in so little time. I now look forward to the expansion of clinical genetics services in Auckland and elsewhere in New Zealand, the establishment of a family clinic for hereditary bowel (and other) cancer, and returning once again to my microscope.

Department of Pathology,

J R JASS

University of Auckland School of Medicine,

Private Bag 92019, Auckland,

New Zealand 